\title{
Gaze cues influence the allocation of attention in natural scene viewing
}

\author{
Stephen R. H. Langton ${ }^{1}$, Christopher O'Donnell ${ }^{2}$, Deborah M. Riby ${ }^{1}$ \\ \& Carrie J. Ballantyne ${ }^{1}$ \\ ${ }^{1}$ University of Stirling, Stirling, UK \\ and \\ ${ }^{2}$ University of Paisley, Paisley, UK
}

Short Title: Gaze cues in natural scene viewing

Address correspondence to: Dr. Stephen R. H. Langton, Department of Psychology, University of Stirling, Stirling, FK9 4LA, UK

Email: srhl1@stirling.ac.uk

Phone: (+44) (0)1786 467659

Fax: (+44) (0)1786 467641 


\begin{abstract}
In two experiments we examined whether the allocation of attention in natural scene viewing is influenced by the gaze cues (head and eye direction) of an individual appearing in the scene. Each experiment employed a variant of the flicker paradigm in which alternating versions of a scene and a modified version of that scene were separated by a brief blank field. In Experiment 1, participants were able to detect the change made to the scene sooner when an individual appearing in the scene was gazing at the changing object than when the individual was absent, gazing straight ahead or gazing at a non-changing object. In addition, participants' ability to detect change deteriorated linearly as the changing object was located progressively further from the line of regard of the gazer. Experiment 2 replicated this change detection advantage of gaze-cued objects in a modified procedure using more critical scenes, a forced choice change/no-change decision and accuracy as the dependent variable. These findings establish that in the perception of static natural scenes and in a change detection task, attention is preferentially allocated to objects that are the target of another's social attention.
\end{abstract}


One of the goals of research in scene perception is to identify the factors that control how visual attention is deployed within an image of a complex natural scene. Research has concentrated on how low-level properties of the visual image can influence attention and how strategies and intentions of the viewer can modulate or override these image-based factors (e.g., Henderson, 2003). However, natural scenes often contain other human beings whose social signals may well serve to indicate important aspects of the environment which might be deserving of immediate scrutiny by a viewer's visual system. The goal of the present paper is therefore to establish whether the eye-gaze and head orientation of someone appearing in a scene can influence the deployment of an observer's attention within the image of that scene. We did this by exploiting the change blindness phenomenon which, Rensink (2000) has argued, can tell us a great deal about the way we scrutinise natural scenes and the nature of our representations of those scenes.

A large body of recent experimental work has established that, under certain circumstances, human observers have great difficulty in detecting even quite large changes made to visible features in natural scenes. For example, in the flicker paradigm, introduced by Rensink, O'Regan \& Clarke (1997), participants are presented with alternating versions of a scene and a modified version of that scene. These modifications might include fairly large changes such as the removal of an object, a change in colour or location of an object, or the substitution of an object with another object. Ordinarily the motion transients produced by these changes make them trivially easy to detect and report. However, if the two versions of the scene are separated by the presentation of a brief blank interval observers take a surprisingly long time to spot these changes. This so-called change blindness effect has also been noted when the change to the scene occurs during a saccadic eye-movement (e.g., McConkie \& Currie, 1996), a film cut (Levin \& Simons, 1997) or when the change occurs at 
the same time as small local transients such as "mudsplashes" are presented on the display (O'Regan, Rensink \& Clarke, 1999).

Some have used these observations to challenge the notion that we build up and retain a rich, stable, detailed representation of a scene (e.g., Rensink, 2000; Rensink et al., 1997; Simons \& Levin, 1997) whilst others have argued that a detailed representation of a scene $i s$ built up, and that change blindness occurs either because of a difficulty in retrieving information from the original scene or a difficulty in comparing this information with that encoded from the changed scene (Angelone, Levin \& Simons, 2003; Hollingworth \& Henderson, 2002; Hollingworth, 2003). Common to both retention failure and retrieval/comparison failure explanations of change blindness is the need for spatial attention to detect change, either through creating a "coherent" object representation (Rensink, 2000) which can survive a visual disruption, or by supporting retrieval from memory of recently encoded object representations at the attended location (Hollingworth, 2003; Hollingworth \& Henderson, 2002). Given this emphasis on spatial attention in scene perception and change detection it becomes important to understand the factors which affect its allocation in natural scenes. To this end, studies of eye-movement control in scene perception have suggested that fixations in a scene, including the first, are allocated to "interesting and informative" scene regions (see Henderson, 2003, for a recent review). Henderson (2003) argues that these interesting and informative regions are determined by bottom-up, stimulus-based factors such as edge density and local contrast; and top-down, memory-based knowledge including episodic knowledge of a particular scene, generic knowledge about types of scenes and taskrelated knowledge. However, there may well be other kinds of priorities which will override these factors. If, for example, the goal of scene perception is to establish a scene's "meaning", then the appearance or actions of any individuals who happen to be visible in the scene may become at least as important as establishing whether or not a toaster is present in a scene you 
have determined to be a kitchen. The suggestion is therefore that certain socially significant stimuli might also influence the allocation of attention in natural scenes.

Several lines of research have suggested that faces themselves (e.g., Ro, Russell \& Lavie, 2001) and angry facial expressions (e.g., Eastwood, Smilek \& Merikle, 2001; Fox, Lester, Russo, Bowles, Pichler \& .Dutton, 2000) are able to influence the allocation of an observer's attention, although natural scenes were not used in these experiments. A similar bias towards the eyes has been suggested by a study conducted by Smilek, Birmingham, Cameron, Bishoff and Kingstone (2006) which did employ natural scenes. In this study, participants whose eye movements were recorded when viewing the real-world scenes tended to make saccades towards the eyes of people in the scenes rather than to other objects or background regions of the scenes. Attention might be biased towards the eyes because they carry important information about face identity (Sadr, Jarudi \& Sinha, 2003) and dispositional states (BaronCohen, 1995); however, the eyes, along with head orientation and body posture, also provide clues as to the target of another individual's attention which could well form an additional "interesting and informative" scene region which might be expected to attract a viewer's visual attention. Indeed, studies using a version of Posner's (1980) pre-cueing paradigm have suggested that cues such as eye-gaze direction and head orientation trigger reflexive shifts in an observer's visual attention (Friesen \& Kingstone, 1998; Langton \& Bruce, 1999). However, as with the majority of studies exploring attention capture by faces and threatening facial expressions, the stimuli used in these precuing experiments were never presented in the context of natural scenes; in all cases, participants saw a single, isolated face cue at fixation followed by a letter target in an otherwise empty display. It is therefore far from clear whether, with many other stimuli to compete for attention, social attention cues will continue to initiate an orienting response when viewed in a natural scene (see also Smilek et al., 2006). 
Therefore, the aim of the present studies was to determine whether social attention cues influence the allocation of attention when viewing natural scenes. More specifically, we used a version of the flicker paradigm to examine whether changes made to gazed-at objects in natural scenes were detected sooner (Experiment 1) and more accurately (Experiment 2) than changes to objects that were not the target of another's attention.

\section{Experiment 1}

In Experiment 1, participants were asked to perform a change detection task on a series of nine different scenes where changes were made across a brief blank display. The original and modified version of each scene was alternated until the participant successfully detected and identified the change. Of the nine scenes seen by each participant, only one was critical: for participants in the "cued" condition, the changing object in the critical scene was gazed at by a person who also appeared in the scene; for those in the "neutral" condition, the person in the critical scene stared straight ahead so as not to cue any of the objects in the scene; and in the "absent" condition, a change occurred in the absence of an individual in the scene. If attention is allocated to gazed-at locations in a natural scene, we would expect changes to be detected sooner in the cued scene than in either the neutral or absent scenes. Furthermore, in order to establish whether or not attention might be drawn to the precise target of another's attention we also included a number of "uncued" conditions. In these, the changing object appeared at increasingly larger angles from the gazer's line of regard.

\section{Method}

\section{Participants}

Three hundred and sixty undergraduate university students from the Universities of Glasgow and Strathclyde took part in return for payment. All had normal or corrected-tonormal vision. 


\section{Apparatus and Materials}

The critical items in this experiment were three natural scenes, photographed using a digital camera and edited using Adobe Photoshop on an Apple Macintosh G3 computer. Each scene was a full-colour image measuring $18 \mathrm{~cm}$ by $13.5 \mathrm{~cm}$ and was themed around a typical work environment (an office, a store room and a common room). At test, these images were viewed from a distance of $0.7 \mathrm{~m}$ on an Apple 21 inch monitor driven by a Apple Macintosh G3 computer running SuperLab software (Cedrus Corps). Several different versions of each scene were prepared, the majority of which contained a gazer who always appeared in the centre of the scene with their body oriented straight towards the camera but with their head and eyes directed along one of six different lines: horizontally towards their left $\left(0^{\circ}\right)$, and then along lines radiating anti-clockwise from this line at $45^{\circ}, 135^{\circ}, 180^{\circ}$ (i.e., horizontally towards their right), $225^{\circ}$, and $315^{\circ}$. Another version of each scene was created with the gazer looking directly towards the camera (to be used in the neutral condition), and another with the gazer removed from the scene (the absent condition). 


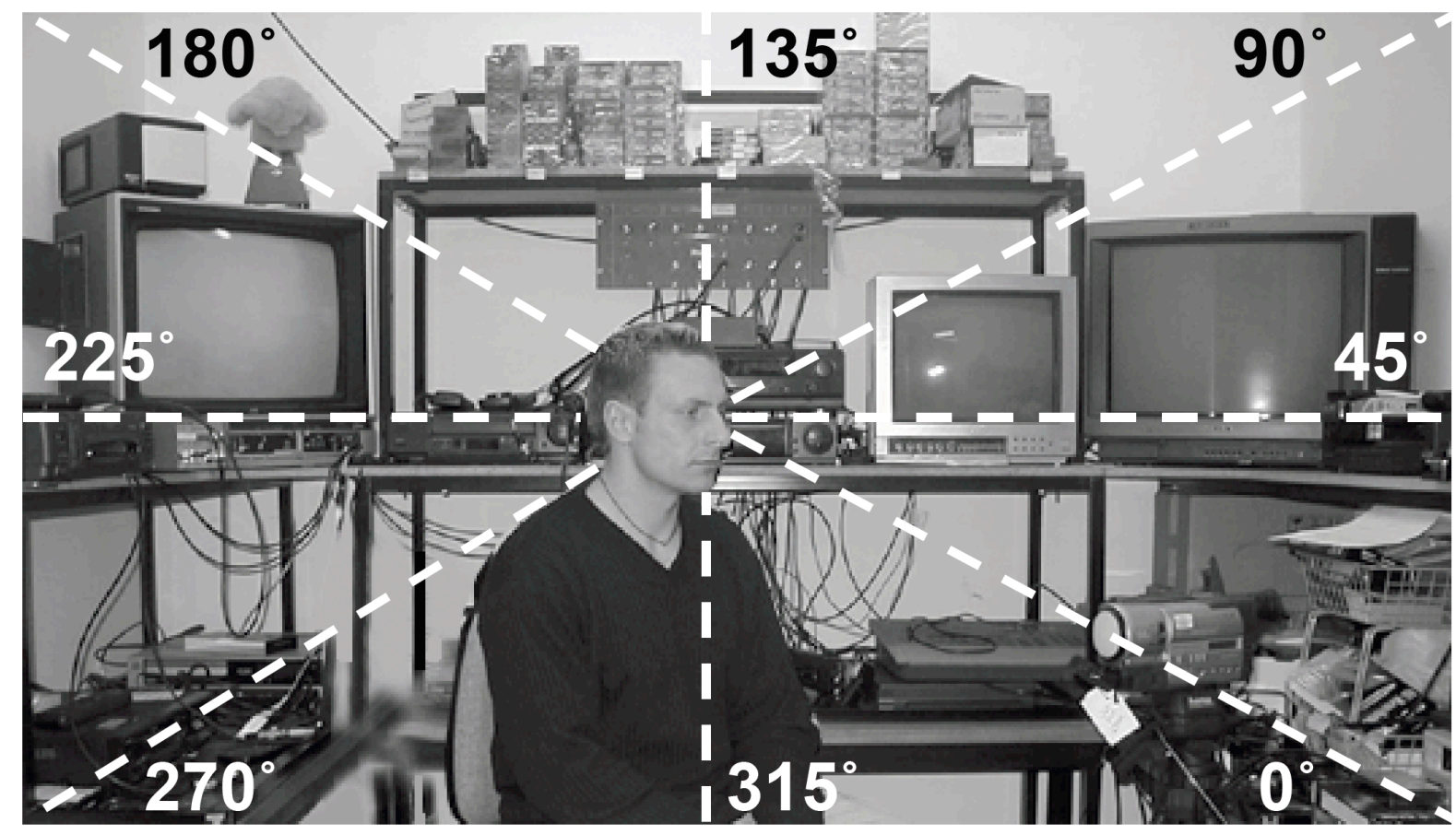

Figure 1. An illustration of the locations of target objects relative to the line of regard of the gazer in Experiment 1. Here the model is gazing along the $0^{\circ}$ line at the camcorder which would be the changing object in the "cued" condition. Changes in other conditions could be made to objects located along the other lines as indicated.

For each of the three critical scenes and each of the six gaze directions, eight different changed scenes were created. These changed scenes were identical to the original versions save for the removal of a single object such as a vase, a bottle of water or a handbag. One change was made to an object along the horizontal line to the gazer's right $\left(0^{\circ}\right)$, another to an object located along a line radiating $45^{\circ}$ anticlockwise from the horizontal and then others along lines radiating anti-clockwise $90^{\circ}, 135^{\circ}, 180^{\circ}, 225^{\circ}, 270^{\circ}$, and $315^{\circ}$ from this line. So, for each gaze direction a change was made to a gazed-at or cued object and then to objects located at lines radiating $45^{\circ}, 90^{\circ}, 135^{\circ}, 180^{\circ}, 225^{\circ}, 270^{\circ}$, and $315^{\circ}$ from the gazer's line of regard (see Figure 1). The same object changes at the identical locations were also made to the absent and neutral scenes. In all cases the to-be-removed objects were placed as far as possible along the lines towards the outer edges of each of the three critical scenes. In this way we hoped to ensure that all changes were set in the margins of interest in the scene 
(Rensink et al., 1997). Indeed, each of 12 independent judges shown all of the changes judged them as being of marginal rather than central interest in the scene.

In addition to these critical scenes, eight "filler" scenes were created. These were the same size as the critical scenes and all contained several people; for example, one scene depicted a dinner party, another a street scene. As with the critical scenes, changed versions of each of these filler scenes were created by removing objects from the scenes using Adobe Photoshop. Care was taken to ensure that in none of these scenes were the changing objects gazed at by people in the scenes.

\section{Design and Procedure}

The materials were tested in a between-subjects design with each participant assigned to one of 10 possible conditions: eight of these were related to the angle subtended at the gazer's face between the location of the manipulation and their line of regard $\left(0^{\circ}\right.$ [i.e., cued], $45^{\circ}, 90^{\circ}$, $135^{\circ}, 180^{\circ}, 225^{\circ}, 270^{\circ}, 315^{\circ}$ ), and the remaining two were a neutral gaze condition, where the gazer looked directly out of the scene towards the viewer, and an absent condition where the gazer did not appear in the scene. Thirty-six participants took part in each of the 10 conditions with each critical scene being seen by 12 participants per condition. For each critical scene in the $0^{\circ}$ (cued) condition, two participants saw a gaze at $0^{\circ}$ and a change at this location, two participants saw a gaze at $45^{\circ}$ and change at $45^{\circ}$ and so on for each of the six gaze directions. For the neutral and absent conditions, the changes always occurred at these same six locations and, for each critical scene, a change at each location was seen by two participants. In each of the remaining (uncued) conditions, each critical scene was again viewed by 12 participants; two of these saw a scene with a gaze at $0^{\circ}$ (i.e., towards the centre right of the picture) and a change at the location defined by the particular uncued condition (e.g., at $45^{\circ}$ anticlockwise from the gazed-at location for the $45^{\circ}$ condition, at $90^{\circ}$ from the gazed-at location for the $90^{\circ}$ 
condition etc.), two saw a gaze at $45^{\circ}$ (i.e., the top right of the picture) and a change at the appropriate location (e.g., at the $90^{\circ}$ [top centre] location for the $45^{\circ}$ condition, at the $135^{\circ}$ [top left] location for the $90^{\circ}$ condition etc.) and so on for each of the six gaze locations. This meant that, although each gaze direction appeared equally often in each condition of the experiment, the subset of change locations was different for each of the uncued conditions. However, it is important to note that change locations were identical for the absent, neutral, cued and $180^{\circ}$ conditions.

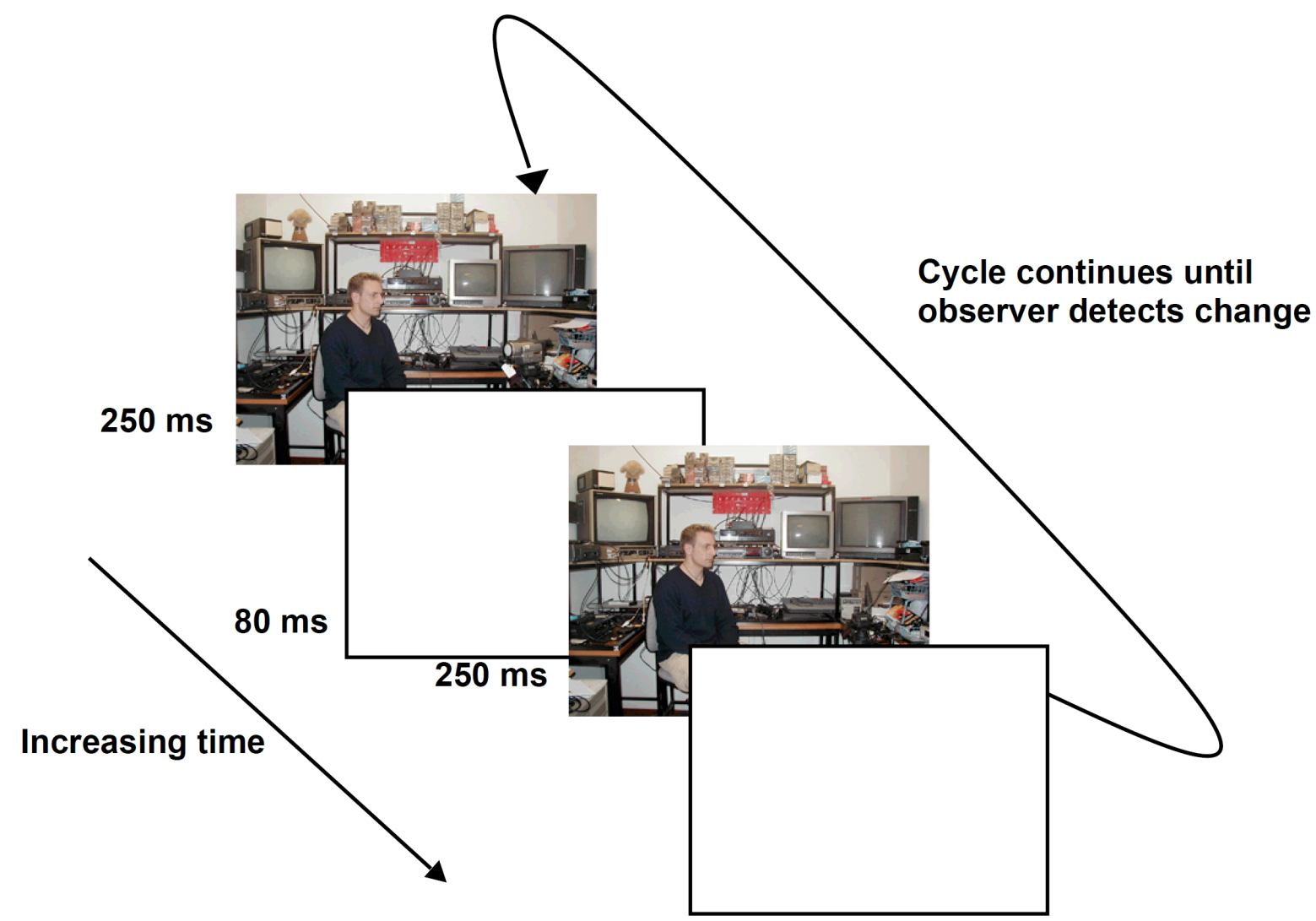

Figure 2. Example of the events in a typical trial in Experiment 1. A trial began with a $250 \mathrm{~ms}$ presentation of the original scene. After an $80 \mathrm{~ms}$ presentation of a blank white screen, the modified version of the scene was displayed, again for $250 \mathrm{~ms}$. A blank screen was then displayed until the participant pressed the space bar which initiated a repeat of this sequence, or the participant correctly identified the change in which case the trial was terminated.

Participants were presented with a single critical scene and the eight filler scenes in a modified version of the flicker paradigm. Each trial in the experiment consisted of an 
alternating sequence of an original image and a modified image separated by a blank white field. A trial began with the presentation of the original image for $250 \mathrm{~ms}$ followed by a blank field for $80 \mathrm{~ms}$ and then the modified version of the scene for $250 \mathrm{~ms}$ (see Figure 2). Following this alternation the scene went blank and remained so until the participant pressed the space bar on a standard keyboard which initiated another alternation of the two images. This sequence was repeated until the participant correctly identified the change or 40 cycles had been repeated in which case the participant's data was discarded. The dependent measure was the number of alternations required to identify the change correctly. The order of presentation of the critical scene and filler scenes was randomised for each participant.

\section{Results and Discussion}

Figure 3 shows the mean number of alternations of the original and modified scenes that participants required in order to identify the changes in each of the experimental conditions. Participants required fewer alternations to identify the changes in the cued condition (mean = 5.9) compared to the absent condition $($ mean $=11.7)$ and were slower still in the neutral condition (mean $=15.2$ ). It appears, therefore, that a cue provided by the model's gaze reduced the number of alternations required to detect a change made to the scene. A betweensubjects ANOVA conducted on the alternations data in cued, absent and neutral conditions confirmed that change detection varied as a function of cue condition, $F(2,105)=36.51, p<$ 0.01. In support of the above observations, Post-hoc Tukey HSD tests $(\alpha=0.05)$ indicated that performance was significantly better in the cued condition compared to both absent and neutral conditions. Moreover, performance was significantly better in the absent compared to the neutral condition. As most prominent theories of change detection suggest that visual attention is necessary to perceive changes in scenes (Rensink, 2000), the implication is therefore that the allocation of participants' attention within a scene is influenced by the social attention signals of individuals appearing in that scene. 


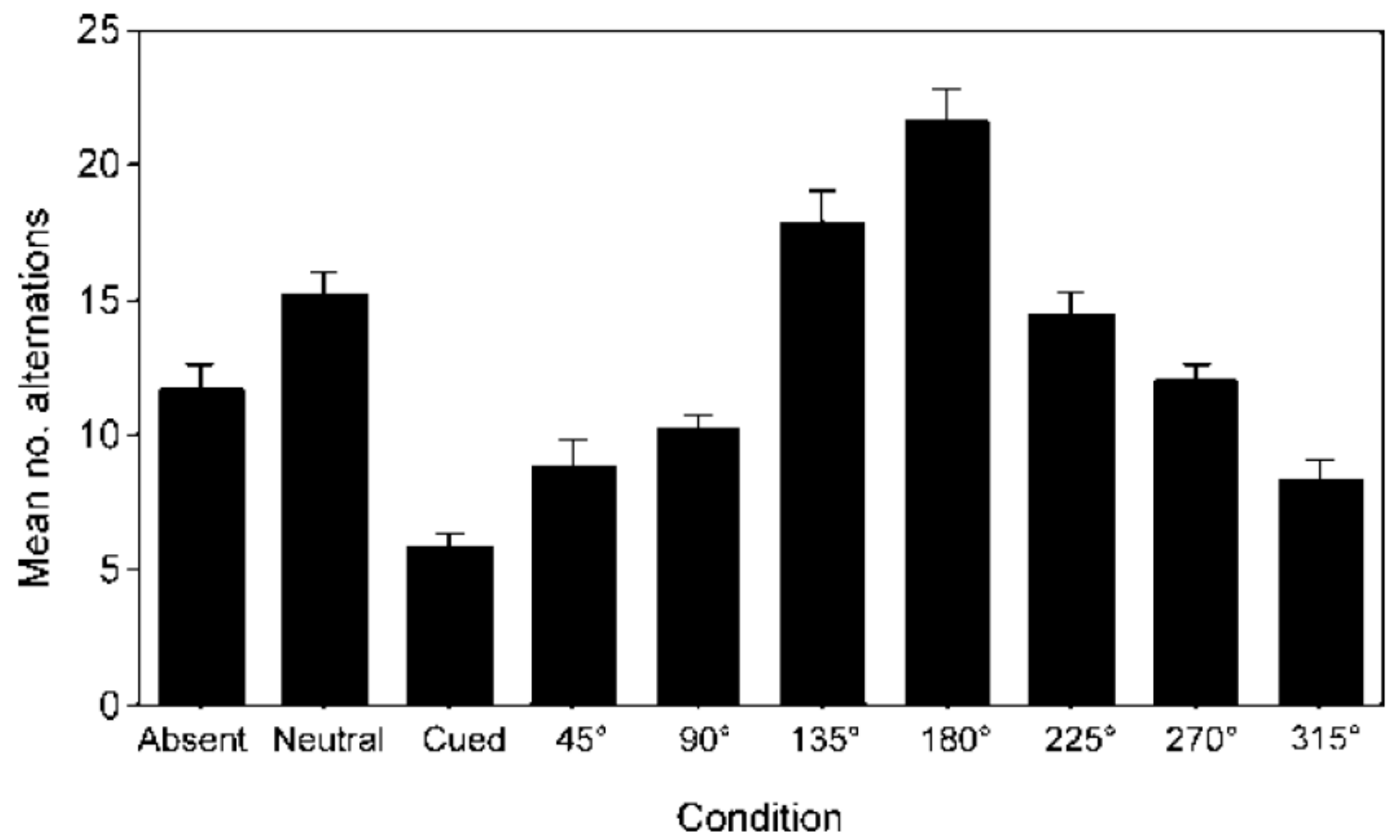

Figure 3. The mean number of alternations required to detect change as a function of condition in Experiment 1. Error bars represent one standard error of the mean in each condition.

Inspection of Figure 3 also suggests that performance deteriorated linearly as the location of the changing object moved from the cued location anticlockwise at increasing angles from the gazer's line of regard until it was located directly behind his head (i.e., $180^{\circ}$ ). Performance then began to recover in the same linear fashion as the location of the changing object re-approached the gazer's line of regard.

In order to test for a linear trend, the data from the target locations at equivalent angles from the gazer's line of regard were first collapsed together (i.e., $45^{\circ}$ and $315^{\circ} ; 90^{\circ}$ and $270^{\circ}$; $135^{\circ}$ and $225^{\circ}$ ) and participants' mean scores in the resulting three conditions (labelled $45^{\circ}$, $90^{\circ}$, and $\left.135^{\circ}\right)$ along with their mean scores in the cued $\left(0^{\circ}\right)$ and $180^{\circ}$ conditions were entered into a trend analysis. Results indicated a significant linear trend, $F(1,283)=256.59, p<0.01$, supporting the observation that performance deteriorated linearly as the target location moved increasingly further from the gazer's line of regard. One interpretation of this finding is that 
attention is initially drawn to the target of the gazer's attention. If this also happens to be the changing object then it will be detected fairly efficiently, either because its representation achieves "coherence" (Rensink, 2000) or because information about the attended location (i.e., that it used to contain an object) can be retrieved from long term memory (Hollingworth \& Henderson, 2002). If no change is detected at the gazed-at location, then the viewer begins to search the scene systematically for the change, beginning with locations adjacent to the gazed-at object followed by locations immediately adjacent to these, and so on through the rest of the scene. Whether or not search proceeds in this manner, it is clear from this experiment that changes were detected at the cued location before changes at any of the other locations.

One potential limitation of Experiment 1 is that only three scenes were used as the critical stimuli. Although the main findings were found to be equivalent across these scenes, it is nevertheless possible that some idiosyncratic property of these scenes contributed to the effect. This issue was addressed in Experiment 2.

\section{Experiment 2}

Across the whole of Experiment 2, participants were exposed to a total of 36 critical scenes which appeared in cued, neutral and uncued conditions and were tested in a withinsubjects design. An additional change from Experiment 1 was the inclusion of a number of no-change trials and instead of allowing participants to repeat alternations of the original and modified scenes until they had identified the change, they were shown just two cycles of these scenes and were asked to decide whether a change had occurred or not. The dependent variable in this experiment was the proportion of trials in which a change was correctly identified. 


\section{Method}

\section{Participants}

Eighteen participants were recruited from the University of Stirling. All had normal, or corrected-to-normal vision.

\section{Apparatus and Materials}

The stimuli were photographed using an Olympus C-900 zoom digital camera and transferred onto PC for use in Adobe Photoshop 5.0. Stimuli were colour photographs of 36 scenes measuring $22.58 \mathrm{~cm}$ by $16.93 \mathrm{~cm}$, with one item in each scene being designated as the target object. Three versions of each scene were created: a "cued" version in which an individual appearing in the scene directed their head and gaze toward the target object, a second "uncued" version in which this individual directed their attention to another, nontarget, object in the scene, and finally a "neutral" version in which the same individual directed their attention towards the camera. The position of the target object was balanced across the scenes, appearing equally often on the left and right side of the scene. The location of the object change with respect to the angle of gaze of the viewer in the uncued condition was not controlled in this experiment. Two different actors were used, each appearing in half of the scenes.

Each of the cued, uncued and neutral scenes was paired with an identical copy of that version of the scene to create the no-change trials. Change trials were created by pairing each version of the scene with a modified version as in Experiment 1. At test, the change and nochange trials were presented using SuperLab software on a Macintosh G4 computer with a 16 inch colour monitor viewed from a distance of $0.7 \mathrm{~m}$. 


\section{Design and Procedure}

In Experiment 2 the cued, neutral and uncued scenes were tested in a within-subjects design with the dependent variable being the proportion of trials in which participants correctly identified that a change had occurred (hit rate).

Each trial in the experiment began with a $250 \mathrm{~ms}$ presentation of the original version of the scene followed by an $80 \mathrm{~ms}$ presentation of a blank field and then a $250 \mathrm{~ms}$ presentation of either the identical scene or the modified version of the scene. A blank screen was then presented for $1000 \mathrm{~ms}$ and then each pair of scenes was presented again in the same way. After this second cycle a blank screen was presented until participants had made their response which they did by pressing the "a" key on a standard keyboard if they thought a change had occurred, or the "l" key if they thought the scenes were unchanged. Participants were asked to respond as accurately as possible and they were told that their speed of response was unimportant. The inter-trial interval was $2500 \mathrm{~ms}$.

Before the experiment, the 36 scenes were randomly divided into three groups of 12 scenes. Within each group, half of the scenes were randomly designated to appear in nochange trials and the other half in change trials. These three groups of scenes were then rotated around conditions for the 18 participants who viewed the scenes in a random order. Prior to the presentation of the 36 trial scenes, participants viewed three practice scenes in which no actor appeared.

\section{Results and Discussion}

The percentage of trials in which each participant correctly responded that a change had occurred was calculated for scenes in each of the three experimental conditions. On average, participants correctly detected changes on $88 \%$ of trials in the cued condition, $53 \%$ of trials in the neutral condition and $44 \%$ of trials in the uncued condition. The average percentage of 
false alarms (trials in which participants wrongly judged that a change had been made) across the whole experiment was just $23 \%$ suggesting that participants were able to discriminate between trials where changes did and did not occur ${ }^{1}$. Thus, as in Experiment 1, participants' ability to spot changes was superior when the changed items were gazed at by someone appearing in the scene than when no information was given as to the location of the changed item. These observations were supported by a repeated measures ANOVA conducted on the accuracy data. This yielded a main effect of condition, $F(2,34)=13.86, p<.01$, and post hoc Tukey HSD tests $(\alpha=0.05)$ confirmed that participants detected targets on a significantly higher percentage of trials in the cued condition than in both the neutral and uncued conditions, which did not differ. Experiment 2 has therefore repeated the findings of Experiment 1 using a modified procedure with a different dependent variable and a withinsubjects design.

\section{General Discussion}

The aim of the experiments reported here was to establish whether cues to another's social attention influence the allocation of an observer's attention when viewing natural scenes. In both experiments participants detected changes made to real world scenes sooner (Experiment 1) and more accurately (Experiment 2) when someone in the scene was directing their attention toward the changing object than when no gaze cue was present or when the individual in the scene was gazing at a non-changing object. These findings suggest that in the perception of static natural scenes, attention is preferentially allocated to objects that are the target of another's social attention, at least when participants are engaged in a change detection task.

Through what mechanism might gaze direction influence the allocation of attention when viewing natural scenes? One possibility is that the social cues signalling attention direction 
are available fairly early in processing and influence the allocation of attention in scenes through largely bottom-up means This could be achieved through an initial allocation of attention to the face (e.g., Ro et al., 2001) - prompted by either its low-level, image-based properties (e.g., high edge density or high local contrast in the eye region) or through its rapid categorisation via dedicated neural circuitry (see Haxby, Hoffman \& Gobbini, 2000, for a review) - followed by an analysis of gaze direction and a subsequent shift of attention in this direction. On the other hand, both faces themselves and their direction of attention might be computed pre-attentively prompting an allocation of attentional resources towards the object at which the face is looking without the need for an initial orienting response toward the face itself. Another possibility is that a priority for attending to whatever faces are looking at might be established through top-down mechanisms. One could imagine, for example, how a kind of default attentional setting to search where faces are looking could be established by providing top-down activation to the network which processes gaze direction. Alternatively, participants may deploy attention rather more strategically to inspect the object of interest in the scene, particularly if they suspect that this will help them complete the task. However, we think this kind of strategic use of attention is unlikely; in Experiment 1, for instance, participants in the cued condition saw just a single cued scene making it unlikely that they learn to adopt this strategy during the course of the experiment.

Clearly the precise mechanism underlying the effect requires further investigation. In addition, it remains to be seen whether the present findings will extend to the perception of dynamic natural scenes where the goals of a perceiver may change from moment to moment dependent on the situational context. Indeed, the study of attention under these real-world circumstances presents a real challenge for cognitive psychology and cognitive neuroscience (see Smilek et al., 2006, for a discussion of this issue). However, the present research clearly establishes that gaze cues appearing in a cluttered visual environment are capable of 
producing an attentional bias such that the object of another's attention is prioritised in the perception of static natural scenes. 


\section{References}

Angelone, B. L., Levin, D. T., \& Simons, D. J. (2003). The relationship between change detection and recognition of centrally attended objects in motion pictures. Perception, 32, 947-962.

Baron-Cohen, S. (1995). Mindblindness: An essay on autism and theory of mind. Cambridge, MA: MIT Press/Bradford Books.

Eastwood, J. D., Smilek, D., \& Merikle, P. M. (2001). Differential guidance by unattended faces expressing positive and negative emotion. Perception \& Psychophysics, 63, 10041013.

Fox, E., Lester, V., Russo, R., Bowles, R. J., Pichler, A., \& Dutton. (2000). Facial expressions of emotion: Are angry faces detected more efficiently? Cognition and Emotion, 14, 61-92.

Friesen, C. K. \& Kingstone, A. (1998). The eyes have it!: reflexive orienting is triggered by nonpredictive gaze. Psychonomic Bulletin and Review, 5, 490-495.

Haxby, J. V., Hoffman, E. A., \& Gobbini, M. I. (2000). The distributed human neural system for face perception. Trends in Cognitive Sciences, 4, 223-233.

Henderson, J. M. (2003). Human gaze control during real-world scene perception. Trends in Cognitive Sciences, 7, 498-504.

Hollingworth, A., \& Henderson, J. M. (2002). Accurate memory for previously attended objects in natural scenes. Journal of Experimental Psychology: Human Perception and Performance, 28, 113-136.

Hollingworth, A. (2003). Failures of retrieval and comparison constrain change detection in natural scenes. Journal of Experimental Psychology: Human Perception and Performance, $29,388-403$.

Langton, S. R. H., \& Bruce, V. (1999). Reflexive visual orienting in response to the social attention of others. Visual Cognition, 6, 541-567.

Levin, D. T., \& Simons, D. J. (1997). Failure to detect changes to attended objects in motion pictures. Psychonomic Bulletin and Review, 4, 501-506. 
McConkie, G. W., \& Currie, C. B. (1996). Visual stability across saccades while viewing complex pictures. Journal of Experimental Psychology: Human Perception and Performance, 22, 563-581.

O’Regan, J. K., Rensink, R. A., \& Clarke, J. J. (1999). Change-blindness as a result of “mudsplashes". Nature, 398, 34.

Posner, M. I. (1980). Orienting of attention. Quarterly Journal of Experimental Psychology, 32, 3-25.

Rensink, R. A. (2000). The dynamic representation of scenes. Visual Cognition, 7, 17-42.

Rensink, R.A., O'Regan, J.K., \& Clark, J.J. (1997) To see or not to see: The need for attention to perceive changes in scenes. Psychological Science, 8, 368-373.

Ro, T., Russell, C., \& Lavie, N. (2001). Changing faces: A detection advantage in the flicker paradigm. Psychological Science, 12, 94-99.

Sadr, J., Jarudi, I., \& Sinha, P. (2003). The role of eyebrows in face recognition. Perception, $32,285-293$

Simons, D. J., \& Levin, D. T. (1997). Change blindness. Trends in Cognitive Sciences, 1, 261-267.

Smilek, D., Birmingham, E., Cameron, D., Bischof, W., \& Kingstone, A. (2006). Cognitive ethology and exploring attention in real-world scenes. Brain Research, 1080, 101-119. 


\section{Footnote}

${ }^{1}$ For trials in which no change occurred there is no distinction between cued and uncued conditions. However, the average percentage of false alarms in trials containing a gaze cue (i.e., cued and uncued trials) was $21 \%$ which did not differ from the average percentage of false alarms in neutral trials $(27 \%, t[17]=1.14, p=0.27)$. 


\section{Author Note}

Correspondence concerning this article should be addressed to Stephen R. H. Langton, Department of Psychology, University of Stirling, Stirling, FK9 4LA, Scotland. Electronic mail may be sent to srhl1@stirling.ac.uk. The authors thank Alan Kingstone and Jari Hietanen for helpful comments on an earlier version of this article. 